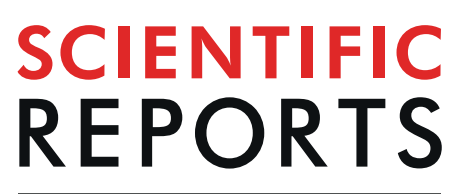

natureresearch

Check for updates

\title{
Shared and Independent Genetic Basis of Resistance to Bt Toxin Cry2Ab in Two Strains of Pink Bollworm
}

\author{
Jeffrey A. Fabrick ${ }^{1 凶}$, Dannialle M. LeRoy ${ }^{1}$, Gopalan C. Unnithan ${ }^{2}$, Alex J. Yelich², \\ Yves Carrière ${ }^{2}$, Xianchun $\mathrm{Li}^{2}$ \& Bruce E. Tabashnik ${ }^{2}$
}

Evolution of pest resistance threatens the benefits of crops genetically engineered to produce insecticidal proteins from Bacillus thuringiensis (Bt). Field populations of the pink bollworm (Pectinophora gossypiella), a global pest of cotton, have evolved practical resistance to transgenic cotton producing Bt toxin Cry2Ab in India, but not in the United States. Previous results show that recessive mutations disrupting an autosomal ATP-binding cassette gene (PgABCA2) are associated with pink bollworm resistance to $\mathrm{Cry} 2 \mathrm{Ab}$ in field-selected populations from India and in one lab-selected strain from the United States (Bt4-R2). Here we discovered that an independently derived, labselected Cry2Ab-resistant pink bollworm strain from the United States (BX-R) also harbors mutations that disrupt PgABCA2. Premature stop codons introduced by mis-splicing of PgABCA2 pre-mRNA were prevalent in field-selected larvae from India and in both lab-selected strains. The most common mutation in field-selected larvae from India was also detected in both lab-selected strains. Results from interstrain crosses indicate BX-R has at least one additional mechanism of resistance to Cry2Ab that does not involve PgABCA2 and is not completely recessive or autosomal. We conclude that recessive mutations disrupting PgABCA2 are the primary, but not the only, mechanism of resistance to Cry2Ab in pink bollworm.

Crops genetically engineered to produce insecticidal proteins from the bacterium Bacillus thuringiensis (Bt) have revolutionized management of some of the world's most devastating pests ${ }^{1}$. In 2018, millions of farmers in 15 countries grew more than 104 million hectares of transgenic Bt crops ${ }^{2}$. Bt crops can suppress pests, increase yield and farmer profits, and decrease conventional insecticide use, which translates to health and environmental benefits ${ }^{1,3-7}$. However, evolution of resistance to Bt crops by pests reduces such benefits ${ }^{8-11}$. Practical resistance to Bt crops, which is field-evolved resistance that has practical consequences for pest management, has been documented in at least nine species of major pests targeted by Bt crops ${ }^{8-11}$.

Better understanding of the mechanisms and genetic basis of resistance to Bt toxins is needed to monitor, manage, and counter pest resistance. For Cryl toxins, the first family of lepidopteran-active crystalline (Cry) Bt toxins deployed widely in transgenic crops, the molecular genetic basis of resistance has been studied extensively ${ }^{12-16}$. However, much less is known about this issue for Cry2 toxins, the second such family. While each of the first Bt crops produced a single toxin from the Cryl family (e.g., Cry1Ac), most Bt crops grown now produce Cry2 $\mathrm{Ab}$ in combination with one or more Cry1 toxins ${ }^{17}$. For lab-selected strains of the lepidopteran pests Helicoverpa armigera and Helicoverpa punctigera, resistance to Cry2 Ab is associated with reduced binding to larval midgut membranes and linked with autosomal recessive mutations that disrupt the ATP-binding cassette protein $\mathrm{ABCA} 2^{18,19}$. Deleting portions of the gene encoding $\mathrm{ABCA} 2$ caused resistance to Cry2 Ab in H. armigera, Trichoplusia ni, and Bombyx mori ${ }^{20-22}$.

Related work with pink bollworm (Pectinophora gossypiella), one of the world's most damaging pests of cotton $^{23,24}$, has provided insights into its lab- and field-selected resistance to Cry2 $\mathrm{Ab}^{25-28}$. The sustained susceptibility to Bt cotton producing Cry1 Ac and Cry2Ab was essential for the recent declaration of eradication of pink bollworm from the continental United States ${ }^{9,29,30}$. Also, populations in China remain susceptible to the 


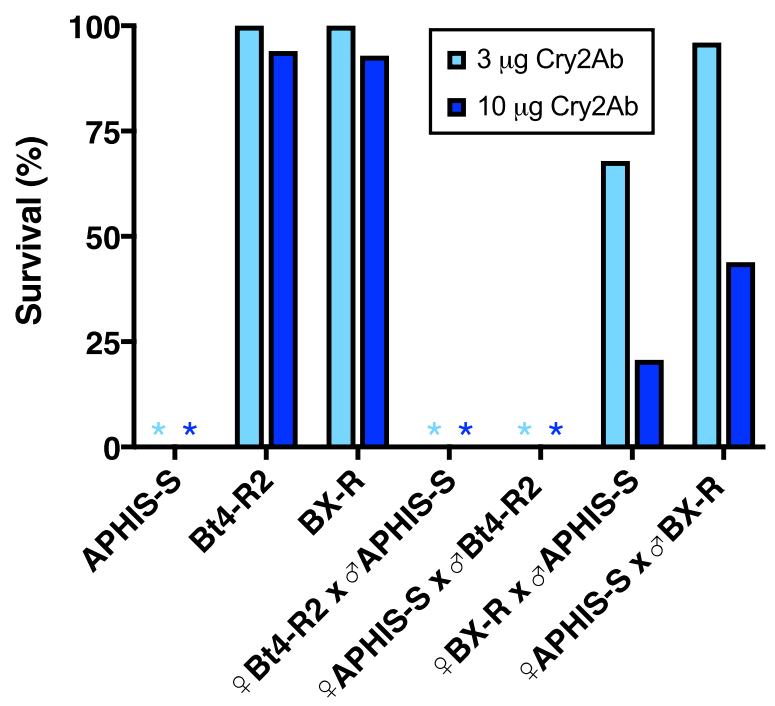

Figure 1. Survival of pink bollworm larvae from two resistant strains (Bt4-R2 and BX-R), a susceptible strain (APHIS-S), and their $\mathrm{F}_{1}$ progeny from mass crosses. Asterisks indicate $0 \%$ survival for APHIS-S and the $\mathrm{F}_{1}$ progeny from the two reciprocal mass crosses between Bt4-R2 and APHIS-S. Light blue: $3 \mu \mathrm{g}$ Cry $2 \mathrm{Ab}$ per $\mathrm{mL}$ $\operatorname{diet}(\mathrm{n}=32$ larvae per bar or asterisk). Dark blue: $10 \mu \mathrm{g}$ Cry2 Ab per $\mathrm{mL}$ diet ( $\mathrm{n}=48$ per bar or asterisk).

CrylAc-producing cotton grown there ${ }^{9,31,32}$. By contrast, pink bollworm in India rapidly evolved resistance to CrylAc and Cry $2 \mathrm{Ab}$ produced by Bt cotton ${ }^{33-35}$. Our previous work revealed that mutations disrupting $P g A B C A 2$, the gene encoding the pink bollworm ABCA2 protein, are associated with resistance to Cry2 Ab in field-selected populations from India and in Bt4-R2, a lab-selected strain from the United States ${ }^{28}$. We previously reported that resistance to Cry $2 \mathrm{Ab}$ was autosomal and recessive in the Bt4-R2 strain ${ }^{27,28}$ and in BX-R, an independently derived lab-selected, strain from the United States ${ }^{25,26}$.

Here we tested the hypothesis that resistance to Cry2Ab in BX-R is associated with mutations in PgABCA2. We conducted interstrain complementation tests for allelism between BX-R and Bt4-R2, and analyzed PgABCA2 cDNA and gDNA from BX-R. We discovered that BX-R harbors disruptive mutations in $P g A B C A 2 \mathrm{cDNA}$, including many novel mutations as well as one reported previously from both India and Bt4-R2, and another reported only from India. The new results also provide evidence that BX-R has a second mechanism of resistance to Cry $2 \mathrm{Ab}$ with inheritance that is not autosomal or recessive.

\section{Results}

Dominance, Sex Linkage and Maternal Effects. In the first and second set of bioassays, respectively, survival at $10 \mu \mathrm{g}$ Cry2 Ab per $\mathrm{mL}$ diet was 95.7 and 92.3\% for Bt4-R2 (mean =94.0\%), 100 and $85.7 \%$ for BX-R (mean $=92.9 \%$ ), and $0 \%$ (both sets) for the susceptible strain APHIS-S (Fig. 1). At $3 \mu \mathrm{g}$ Cry2Ab per mL diet, which was tested only in the first set of bioassays, survival was $100 \%$ for both resistant strains and $0 \%$ for APHIS-S (Fig. 1).

Survival of $\mathrm{F}_{1}$ progeny from mass crosses between Bt4-R2 and APHIS-S was $0 \%$ at 3 and $10 \mu \mathrm{g}$ Cry $2 \mathrm{Ab}$ per $\mathrm{mL}$ diet for both reciprocal crosses, indicating resistance was autosomal and completely recessive ( $h=0$ at both concentrations), with sex linkage and maternal effects not evident (Fig. 1). By contrast, BX-R resistance to Cry $2 \mathrm{Ab}$ was not entirely autosomal or recessive. In the first and second set of bioassays, respectively, at $10 \mu \mathrm{g}$ Cry $2 \mathrm{Ab}$ per $\mathrm{mL}$ diet, survival of $\mathrm{F}_{1}$ progeny from mass crosses was 21.4 and $20.0 \%$ for 9 BX-R X $₫$ APHIS-S ( mean $=20.7 \%$ ) and 44.0 and $43.8 \%$ for 9 APHIS-S X ${ }^{\star} \mathrm{BX}-\mathrm{R}$ (mean $=43.9 \%$, Fig. 1). At $3 \mu \mathrm{g}$ Cry $2 \mathrm{Ab}$ per $\mathrm{mL}$ diet, survival of $\mathrm{F}_{1}$ progeny from mass crosses was $67.9 \%$ for + BX-R X o APHIS-S and $96.0 \%$ for + APHIS-S X $九$ BX-R (Fig. 1). In all three pairwise comparisons between reciprocal crosses (first and second bioassays at $10 \mu \mathrm{g}$ Cry $2 \mathrm{Ab}$ per mL diet and first bioassay at $3 \mu \mathrm{g}$ Cry $2 \mathrm{Ab}$ per $\mathrm{mL}$ diet), survival was higher for $q$ APHIS-S X $\sigma^{\star} \mathrm{BX}-\mathrm{R}$ than for $q \mathrm{BX}-\mathrm{R} \mathrm{X}{ }_{0}$ APHIS-S (mean difference $=25 \%, \mathrm{SE}=2 \%$, paired t-test, $t=14.9, d f=2, P=0.0045$ ).

The higher survival on treated diet of $F_{1}$ progeny from crosses with BX-R fathers versus BX-R mothers is not consistent with maternally inherited resistance, which would be expected to cause the opposite pattern. Although the observed pattern of survival on treated diet is consistent with sex-linked inheritance of resistance, the sex ratio of the survivors is not. Under sex-linked inheritance of resistance, an excess of sons is expected in the $\mathrm{F}_{1}$ survivors on treated diet from crosses with BX-R mothers relative to BX-R fathers (see Methods). However, the proportion of males was not higher for the $\mathrm{F}_{1}$ survivors from crosses with BX-R mothers $(0.54,7$ of 13$)$ than BX-R fathers $(0.85,17$ of 20$)$. On untreated diet, the proportion of $F_{1}$ males did not differ from the expected 0.50 for the crosses with $\mathrm{BX}-\mathrm{R}$ fathers $(0.50,4$ of 8 ) or mothers ( 5 of $8,0.63$, Fisher's exact test, $\mathrm{P}=1.0$ for each type of cross). In this experiment, the proportion of $\mathrm{F}_{1}$ males on untreated diet pooled for both types of cross $(0.56,9$ of 16$)$ was similar to the mean proportion of males on non-Bt cotton in previous studies $\left(0.55, \mathrm{SE}=0.005, \mathrm{n}=4\right.$ studies $\left.^{36-39}\right)$.

Based on the mean survival of $\mathrm{F}_{1}$ progeny at $10 \mu \mathrm{g}$ Cry $2 \mathrm{Ab}$ per $\mathrm{mL}$ diet from the first and second bioassays, the dominance parameter $h$ was 0.22 for the crosses with mothers from BX-R and 0.47 for the crosses with fathers 


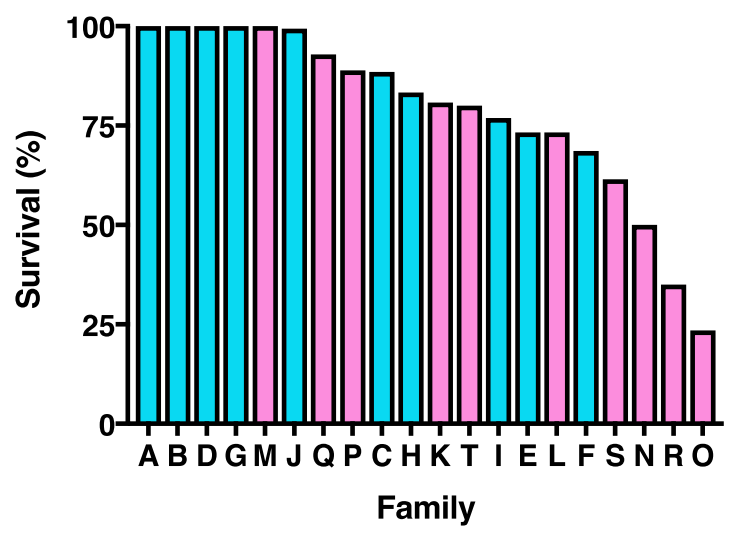

Figure 2. Survival of pink bollworm larvae from single-pair crosses between resistant strains Bt4-R2 and BX-R tested at $10 \mu \mathrm{g}$ Cry2 Ab per mL diet. Families A-J (blue) from female Bt4-R2 X male BX-R and K-T (pink) from female BX-R X male Bt4-R2.

from BX-R (mean $=0.35)$. Based on survival at $3 \mu \mathrm{g}$ Cry2 Ab per $\mathrm{mL}$ diet, $h$ was 0.68 for crosses with BX-R mothers and 0.96 for crosses with BX-R fathers (mean $=0.82$ ). Because $h$ varies from 0 for completely recessive resistance to 1 for completely dominant resistance, these results show that BX-R resistance to Cry2Ab was not completely recessive at either concentration and was close to completely dominant for crosses with BX-R fathers at $3 \mu \mathrm{g}$ Cry $2 \mathrm{Ab}$ per $\mathrm{mL}$ diet.

Interstrain Complementation Test for Allelism. In the $\mathrm{F}_{1}$ progeny of 20 families from single-pair crosses between BX-R and Bt4-R2, mean survival at $10 \mu \mathrm{g}$ Cry2 Ab per $\mathrm{mL}$ diet was $78.8 \%(\mathrm{SE}=5.0 \%)$, which differs significantly from the $0 \%$ survival expected if resistance in both strains was recessive and controlled entirely by loci that differed between strains (one-sample t-test, $t=15.9, d f=19, P<0.0001$ ). The striking heterogeneity in survival among the $20 \mathrm{~F}_{1}$ families (range $=24$ to $100 \%$, Fig. 2) from crosses between BX-R and Bt4-R2 indicates genetic variation affecting resistance occurred within the strains, between the strains, or both. Because resistance was not completely dominant at this concentration in either strain ( $h=0$ in Bt4-R2 and mean $h=0.35$ in BX-R), the results showing six $\mathrm{F}_{1}$ families with survival of $99.4 \%(\mathrm{~J})$ or $100.0 \%(\mathrm{~A}, \mathrm{~B}, \mathrm{D}, \mathrm{G}$, and $\mathrm{M})$ imply that at the same locus in both strains, neither parent for these families had alleles conferring susceptibility to Cry2Ab. Conversely, survival of $<70 \%$ in five families (including $24 \%$ in family O, 35\% in R, and $50 \%$ in $\mathrm{N}$ ) indicates that in these families, the parents from BX-R and Bt4-R2 did not share a locus where both parents had no alleles conferring susceptibility.

Consistent with the results described above showing that inheritance of resistance for $\mathrm{F}_{1}$ progeny from mass crosses between BX-R and APHIS-S was not entirely autosomal, mean survival was $20 \%$ higher for the 10 families with the father from BX-R (A-J: $89 \%, \mathrm{SE}=4 \%)$ than for the 10 families with the mother from BX-R (K-T: $69 \%$, SE $=8.1 \%$, t-test, $t=2.3, d f=9, P=0.041$, Fig. 2 ). Relative to the crosses between BX-R and APHIS-S, mean survival in the crosses between BX-R and Bt4-R2 was 45\% higher for the families with a BX-R father (one-sample $\mathrm{t}$-test, $t=11.3, d f=9, P<0.0001$ ) and $47 \%$ higher for the crosses with the mother from BX-R (one-sample t-test, $t=5.9, d f=9, P=0.0002)$. The significantly higher survival in $\mathrm{F}_{1}$ progeny from crosses between BX-R and Bt4-R2 relative to crosses between BX-R and APHIS-S confirms the two resistant strains shared at least one locus harboring one or more alleles conferring resistance to Cry2 Ab.

PCR Screening of $g D N A$ from BX-R for Mutation $r_{A 1}$ of $P g A B C A 2$. Because previous work identified mutations in PgABCA2 linked with resistance to Cry2Ab in $\mathrm{Bt} 4-\mathrm{R} 2^{28}$, we hypothesized that this is the shared locus where resistance mutations also occur in some individuals from BX-R. In particular, we previously found that mutation $r_{A 1}$, which introduces a premature stop codon in $P g A B C A 2$, occurred in all gDNA screened from Bt4-R2 and in none from APHIS-S ${ }^{28}$. Results in the present study using allele-specific PCR conducted simultaneously for the three strains ( $\mathrm{n}=10$ individuals and 20 alleles per strain) confirmed that the $r_{A 1}$ mutation was fixed in Bt4-R2 (no wild-type) and absent from APHIS-S. The results here also revealed $r_{A 1}$ was absent from $\mathrm{BX}-\mathrm{R}$. Moreover, in $59 \mathrm{~F}_{1}$ survivors on $10 \mu \mathrm{g}$ Cry2 Ab per $\mathrm{mL}$ diet from $20 \mathrm{BX}-\mathrm{R} \mathrm{X} \mathrm{Bt} 4-\mathrm{R} 2$ single-pair families, every individual screened had one $P g A B C A 2$ allele with the $r_{A 1}$ mutation. Because $r_{A 1}$ was detected in all gDNA screened from Bt4-R2 and none from BX-R, we infer that the single allele containing $r_{A 1}$ in each of the $59 \mathrm{~F}_{1}$ survivors came from the Bt4-R2 parent. These results show that $r_{A 1}$ does not confer resistance to Cry2Ab in BX-R, but they do not exclude contributions to Cry2Ab resistance in BX-R from other mutations in PgABCA2.

Mutations in PgABCA2 cDNA and gDNA from BX-R. To determine if BX-R harbored mutations in $P g A B C A 2$ other than $r_{A 1}$, we sequenced cDNA from three clones from each of three BX-R larvae. Confirming the results from the PCR screening of gDNA for the $r_{A 1}$ mutation described above, all nine $P g A B C A 2 \mathrm{cDNA}$ sequences from the three BX-R larvae lacked disruptive indels near exon 20 (Fig. 3 and Table 1) characteristic of the $r_{A 1}$ mutation $^{28}$. However, all nine sequences from BX-R had other mutations affecting the predicted amino acid sequence (Fig. 3, Table 1, Supplementary Fig. S1). Eight of the nine sequences had at least one premature stop codon caused by a deletion (Fig. 3, Table 1, Supplementary Table S1and Supplementary Fig. S1). The exceptional 
a
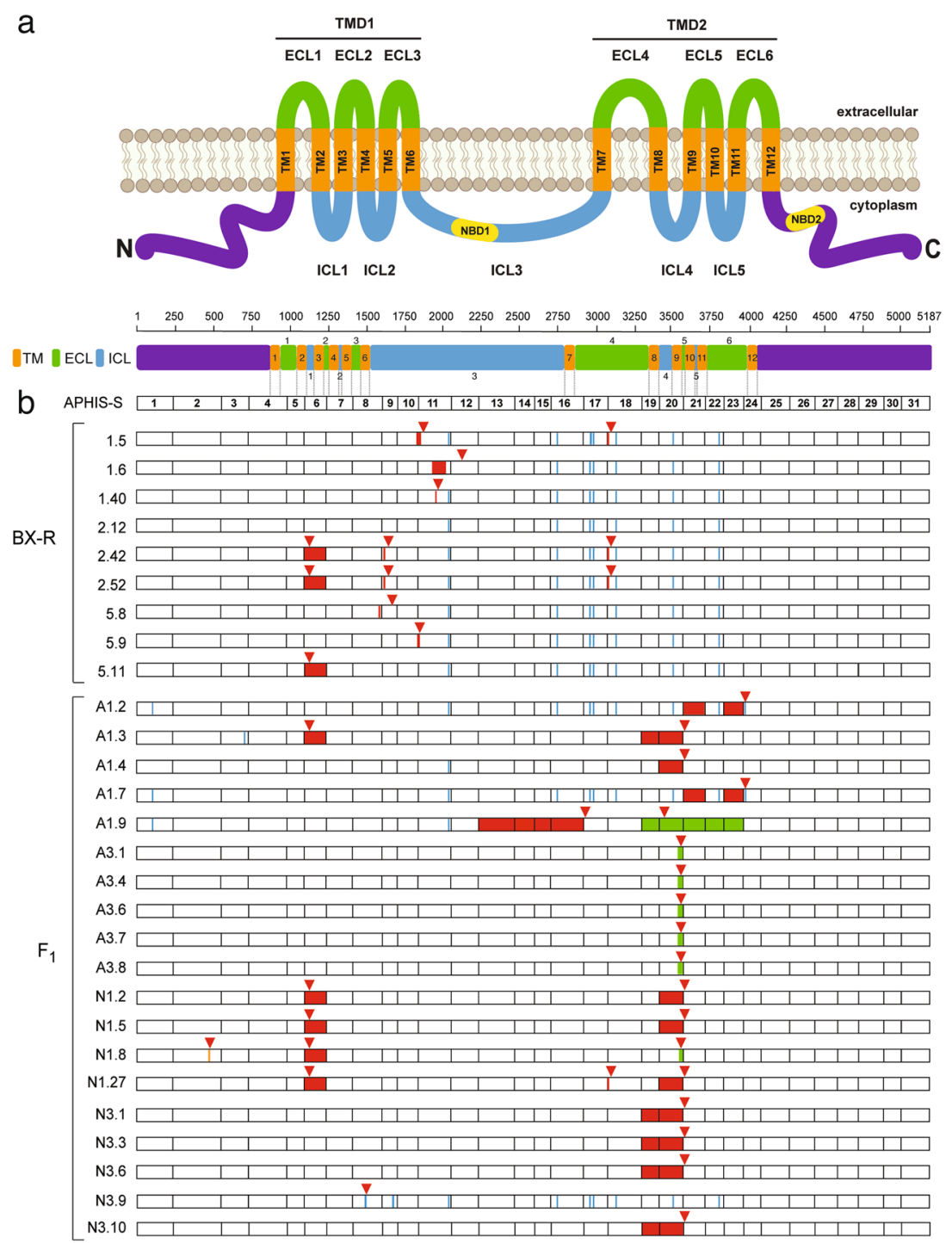

Figure 3. Mutations in $28 \mathrm{PgABCA} 2 \mathrm{cDNA}$ sequences from Cry2Ab-resistant strain $\mathrm{BX}-\mathrm{R}$ and Cry2Abresistant $\mathrm{F}_{1}$ progeny from BX-R X Bt4-R2. (a) The predicted PgABCA2 protein includes amino $(\mathrm{N})$ and carboxyl (C) termini (purple) and two transmembrane domains (TMD1 and TMD2). Each TMD contains six transmembrane-spanning regions (TM, orange), three extracellular loops (ECL, green), and two intracellular loops (ICL, blue). The two TMDs are connected by a single intracellular loop (ICL3). ICL3 and the C-terminal domain each contain a nucleotide-binding domain (NBD, yellow). (b) Mutations in PgABCA2 cDNAs from BX-R (three clones from each of three individuals: 1, 2 and 5) and resistant $F_{1}$ progeny from BX-R X Bt4-R2 (4-5 clones from each of four individuals: A1 and A3 from family A, N1 and N3 from family N, see Fig. 2) relative to the susceptible strain APHIS-S (MG637361). Numbers to the right of the decimal point for each individual indicate the clone sequenced. Exon numbers are shown for APHIS-S. Red triangles above bars indicate premature stop codons, which occur in all sequences except 2.12 from BX-R. Colors within bars show mutations: red for deletions, blue for substitutions, green for insertions and deletions, and orange for the single-bp insertion in exon 2 of N1.8 from the resistant $\mathrm{F}_{1}$ progeny.

sequence was from BX-R larva 2, clone 12 (2.12), which had only amino acid substitutions and no deletions, insertions, or premature stop codons (Supplementary Fig. S1).

Overall, the nine sequences harbored 15 different mutations and a total of 75 mutations counting the multiple occurrences for 10 of the 15 mutations (Table 1). Of the 15 mutations, eight are deletions causing frameshifts that introduce premature stop codons and seven are point mutations that cause single amino acid substitutions (Table 1). Of the seven amino acid substitutions, two are conservative and five are not conservative (E677V, L990S, P992L, H1055N, and S1274G). Each of the eight deletions occurred in at most three sequences from either one or two larvae. By contrast, six of the seven point mutations occurred in all nine sequences and the other occurred in eight of the nine sequences. Only two of the nine sequences were identical; these were from BX-R larva 2, clones 42 and 52 (2.42 and 2.52, Fig. 3, Supplementary Fig. S1). 


\begin{tabular}{|c|c|c|c|c|c|c|}
\hline cDNA mutation & Codon $^{\mathrm{a}}$ & Exon $^{\mathrm{b}}$ & Type $^{\mathrm{c}}$ & Effect $^{\mathrm{d}}$ & In gDNA $^{\mathrm{e}}$ & Larva.clone $^{f}$ \\
\hline c.1090_1234del & 364 & 6 & fs & stop at 373 & No & $2.42,2.52,5.11$ \\
\hline c.1609del & 537 & 8 & fs & stop at 552 & No & 5.8 \\
\hline c.1622_1623del & 541 & 9 & fs & stop at $517^{g}$ & No & \begin{tabular}{|l|}
$2.42,2.52$ \\
\end{tabular} \\
\hline c.1786_1843del & 596 & 10 & fs & stop at 597 & No & 1.5 \\
\hline c.1832_1850del & 611 & 10 & fs & stop at 615 & No & 5.9 \\
\hline c.1905_2031del & 635 & 11 & fs & stop at 662 & No & 1.6 \\
\hline c.1963del & 655 & 11 & fs & stop at $664^{\mathrm{g}}$ & No & 1.40 \\
\hline c.3097_3100del & 1033 & 18 & fs & stop at $1027^{\mathrm{g}, \mathrm{h}}$ & No & $1.5,2.42,2.52$ \\
\hline c. $2030 \mathrm{~A}>\mathrm{T}$ & 677 & 11 & $\mathrm{~ms}$ & E677V & Yes & All but 1.6 \\
\hline c. $2753 \mathrm{G}>\mathrm{C}$ & 918 & 16 & $\mathrm{~ms}$ & C918S & Yes & All \\
\hline c. $2969 \mathrm{~T}>\mathrm{C}$ & 990 & 17 & $\mathrm{~ms}$ & L990S & Yes & All \\
\hline c. $2975 \mathrm{C}>\mathrm{T}$ & 992 & 17 & ms & P992L & Yes & All \\
\hline c. $3163 \mathrm{C}>\mathrm{A}$ & 1055 & 18 & $\mathrm{~ms}$ & H1055N & Yes & All \\
\hline$c .3517 \mathrm{G}>\mathrm{A}$ & 1173 & 20 & $\mathrm{~ms}$ & V1173I & Yes & All \\
\hline c. $3820 \mathrm{~A}>\mathrm{G}$ & 1274 & 22 & $\mathrm{~ms}$ & S1274G & Yes & All \\
\hline
\end{tabular}

Table 1. Fifteen cDNA mutations in PgABCA2 in three Cry2Ab-resistant larvae from the BX-R strain. ${ }^{a}$ Codon where the mutation occurs in the full-length PgABCA2 cDNA sequence (MG637361.1). ${ }^{b}$ Exon where the mutation occurs. ${ }^{c} \mathrm{fs}$, frameshift caused by deletion; ms, missense (point mutation, single amino acid

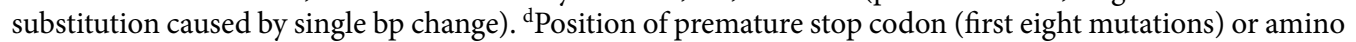
acid substitution (last seven mutations). ${ }^{e}$ Indicates if cDNA mutation also occurs in corresponding gDNA. ${ }^{\text {fLarva }}(1,2$ or 5$)$ and clone where mutation occurred (e.g., 2.42 indicates larva 2, clone 42); three clones were sequenced from each larva (total of nine clones). ${ }^{\mathrm{g}} \mathrm{Affected}$ codons in the mutant and wild-type sequence (from "Codon" column) do not match because additional mutations occur upstream of this mutation. hremature stop codon at 1027 in 1.5; at 997 in 2.42 and 2.52 because of deletion upstream of c.3097_3100del.

Sequencing of gDNA fragments corresponding to each of the 15 PgABCA2 cDNA mutations from $\mathrm{BX}-\mathrm{R}$ revealed that all eight point mutations, but none of the seven deletions, occurred in gDNA (Table 1, Supplementary Table S2). Mutation c1090_1234del is a 145-bp deletion that causes the complete loss of exon 6 because of mis-splicing (Mathew et al. 2018) ${ }^{28}$. Mutations c.1786_1843del, c.1832_1850del, and c.3097_3100del do not cause complete loss of any exons, but are at or span predicted exon-intron junctions and are therefore probably caused by mis-spliced pre-mRNA. Although not precisely located at exon-intron junctions, mutations c.1609del, c.1622_1623del, and c.1963del are within 3-8 bp of their respective splice junctions and could have resulted from incorrect mRNA splicing. The c.1905_2031del mutation is a 127-bp deletion in cDNA not reflected in gDNA that occurs $72 \mathrm{bp}$ upstream of the exon 11 junction and $31 \mathrm{bp}$ downstream of the exon 12 junction. The cause of this deletion is unknown.

Mutations in PgABCA2 cDNA from Cry2Ab-resistant $\mathrm{F}_{1}$ Progeny of BX-R X Bt4-R2 Single-Pair Crosses. We sequenced $P g A B C A 2$ cDNA from 19 clones from four resistant $\mathrm{F}_{1}$ progeny of BX-R X Bt4-R2 single-pair crosses that survived exposure to $10 \mu \mathrm{g}$ Cry2 Ab per $\mathrm{mL}$ diet (larvae A1, A3, N1, and N3 from families A and N, Fig. 2; 4 clones from N1 and 5 clones from each of the other larvae, Fig. 3). All 19 cDNA clones sequenced from these four Cry2Ab-resistant larvae had at least one premature stop codon introduced by a frameshift mutation (Fig. 3, Table 2, and Supplementary Fig. S3).

Overall, the 19 sequences from the resistant $F_{1}$ progeny harbored 22 different mutations and a total of 59 mutations counting the multiple occurrences for 15 of the 22 mutations (Table 2). Of the 22 different mutations in the resistant $\mathrm{F}_{1}$ progeny, twelve were previously identified from BX-R, Bt4-R2, or both; including seven point mutations and one new deletion in BX-R discovered here (Fig. 3, Tables 1 and 2) and four mutations in Bt4-R2 reported by Mathew et al. (2018) ${ }^{28}$ (Fig. 3, Table 2). Only one mutation (c.1090_1234del) was found previously in both BX-R and Bt4-R2 (Fig. 3, Table 2). Ten mutations in the resistant $\mathrm{F}_{1}$ progeny were not found previously in either BX-R or Bt4-R2 (Fig. 3, Table 2). Nine of the 22 mutations in the resistant $\mathrm{F}_{1}$ progeny probably reflect mis-splicing (Table 2, Fig. 3, Supplementary Table S3 and Supplementary Fig. S3). Whereas five of these nine mutations were caused by exon skipping, one involved alternative $5^{\prime}$ and $3^{\prime}$ splice sites, one involved an alternative $3^{\prime}$ splice site, and two indel mutations were caused by intron retention (Table 2, Supplementary Fig. S3). Mutation c.3097_3100del, which was also found in BX-R and in a Cry2Ab-resistant larva (KT-1) from India ${ }^{28}$, is caused by the alternative use of a $3^{\prime}$ splice site (Table 2, Supplementary Fig. S3).

\section{Discussion}

We found that two independently derived, lab-selected strains of pink bollworm, BX-R and Bt4-R2, share a mechanism of resistance to Cry2 $\mathrm{Ab}$, and at least one additional mechanism of resistance occurs in BX-R. The results here confirm our previous findings that disruptive mutations in the $\mathrm{ABC}$ transporter gene $P g A B C A 2$ are associated with the autosomal, recessive resistance of Bt4-R2 to Cry2 $\mathrm{Ab}^{28}$. We discovered in this study that resistance of $\mathrm{BX}-\mathrm{R}$ to Cry2Ab is also associated with disruptive mutations in PgABCA2. We previously found that mutations disrupting PgABCA2 are also associated with resistance to Cry2Ab in field-selected populations of pink bollworm 


\begin{tabular}{|c|c|c|c|c|c|c|c|}
\hline Previous $^{\mathrm{a}}$ & cDNA mutation & Codon $^{\mathrm{b}}$ & Exon $^{c}$ & Type $^{\mathrm{d}}$ & Effect $^{\mathrm{e}}$ & In $_{\text {gDNA }^{f}}$ & Larva.cloneg \\
\hline Neither & c.432_433insT & 144 & 2 & fs & stop at 145 & Yes & $\mathrm{N} 1.8$ \\
\hline Both & c.1090_1234del & 364 & 6 & fs & stop at 373 & $\mathrm{No}^{\mathrm{h}}$ & $\mathrm{A} 1.3, \mathrm{~N} 1.2, \mathrm{~N} 1.5, \mathrm{~N} 1.8, \mathrm{~N} 1.27$ \\
\hline Neither & c.2230_2930del & 744 & $13-16$ & fs & stop at 751 & $\mathrm{No}^{\mathrm{i}}$ & A1.9 \\
\hline BX-R & c.3097_3100del & 1033 & 18 & fs & stop at $997 \mathrm{j}$ & $\mathrm{No}^{\mathrm{k}}$ & $\mathrm{N} 1.27$ \\
\hline $\mathrm{Bt} 4-\mathrm{R} 2$ & c.3313_3589del & 1105 & $19-20$ & fs & stop at $1112 \mathrm{j}, \mathrm{l}$ & $\mathrm{No}^{\mathrm{h}}$ & A1.3, N3.1, N3.3, N3.6, N3.10 \\
\hline Neither & c.3313_3967delins ${ }^{\mathrm{m}}$ & 1106 & $19-23$ & fs & stop at $897 j$ & $\mathrm{No}^{\mathrm{n}}$ & A1.9 \\
\hline Bt4-R2 & c.3418_3589del & 1140 & 20 & fs & stop at $1147^{\circ} \mathrm{O}$ & $\mathrm{No}^{\mathrm{h}}$ & A1.4, N1.2, N1.5, N1.27 \\
\hline Bt4-R2 & c.3556_3588delins ${ }^{\mathrm{m}}$ & 1186 & 20 & fs & stop at $1220 \mathrm{j}, \mathrm{p}$ & $\mathrm{No}^{\mathrm{n}}$ & A3.1, A3.4, A3.6, A3.7, A3.8, N1.8 \\
\hline Neither & c.3840_3967del & 1280 & 23 & fs & stop at 1291 & $\mathrm{No}^{\mathrm{h}}$ & $\mathrm{A} 1.2, \mathrm{~A} 1.7$ \\
\hline Neither & c. $1454 \mathrm{C}>\mathrm{A}$ & 485 & 8 & ns & stop at 485 & Yes & N3.9 \\
\hline Neither & c. $3972 \mathrm{~A}>\mathrm{T}$ & 1325 & 24 & ns & stop at $1240 \mathrm{j}$ & Yes & A1.2, A1.7 \\
\hline Neither & c.3590_3715del & 1197 & 21 & InF & loss of 42 aa & $\mathrm{No}^{\mathrm{h}}$ & $\mathrm{A} 1.2, \mathrm{~A} 1.7$ \\
\hline Neither & c. $101 \mathrm{C}>\mathrm{T}$ & 34 & 1 & $\mathrm{~ms}$ & T34M & Yes & A1.2, A1.7, A1.9 \\
\hline Neither & c. $728 \mathrm{~A}>\mathrm{T}$ & 243 & 3 & $\mathrm{~ms}$ & D243V & Yes & A1.3 \\
\hline Neither & c. $1702 \mathrm{C}>\mathrm{A}$ & 568 & 9 & ms & Q568K & Yes & N3.9 \\
\hline BX-R & c. $2030 \mathrm{~A}>\mathrm{T}$ & 677 & 11 & $\mathrm{~ms}$ & E677V & Yes & A1.2, A1.4, A1.7, A1.9, N3.9 \\
\hline BX-R & c. $2753 \mathrm{G}>\mathrm{C}$ & 918 & 16 & $\mathrm{~ms}$ & C918S & Yes & A1.2, A1.7, N3.9 \\
\hline BX-R & c. $2969 \mathrm{~T}>\mathrm{C}$ & 990 & 17 & ms & L990S & Yes & A1.2, A1.7, N3.9 \\
\hline BX-R & c. $2975 \mathrm{C}>\mathrm{T}$ & 992 & 17 & $\mathrm{~ms}$ & P992L & Yes & A1.2, A1.7, N3.9 \\
\hline BX-R & c. $3163 \mathrm{C}>\mathrm{A}$ & 1055 & 18 & $\mathrm{~ms}$ & H1055N & Yes & A1.2, A1.7, N3.9 \\
\hline BX-R & c. $3517 \mathrm{G}>\mathrm{A}$ & 1173 & 20 & ms & V1173I & Yes & A1.2, A1.7, N3.9 \\
\hline BX-R & c. $3820 A>G$ & 1274 & 22 & $\mathrm{~ms}$ & S1274G & Yes & A1.2, A1.7, N3.9 \\
\hline
\end{tabular}

Table 2. Twenty-two cDNA mutations in PgABCA2 in four Cry2Ab-resistant $\mathrm{F}_{1}$ larvae from BX-R X Bt4-R2. ${ }^{\mathrm{a}} \mathrm{CDNA}$ mutation found previously in BX-R (Table 1), Bt4-R2 (Mathew et al. 2018), both, or neither. ${ }^{\mathrm{b}}$ Codon where the mutation occurs in the full-length PgABCA2 cDNA sequence (MG637361.1). 'Exon where the mutation occurs. ${ }^{\mathrm{d}} \mathrm{fs}$, frameshift caused by deletion; ns, nonsense mutation (single base-pair substitution that introduces premature stop codon); InF, in-frame deletion; ms, missense (point mutation, single amino acid substitution caused by single bp change). ePosition of premature stop codon (first 11 mutations), amino acids

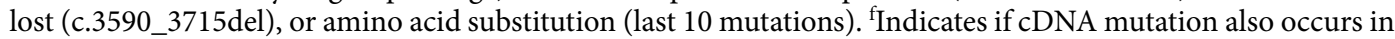
corresponding gDNA. ' ${ }^{\mathrm{L}}$ arva $(\mathrm{A} 1, \mathrm{~A} 3, \mathrm{~N} 1$, or N3) and clone where mutation occurred (e.g., N1.8 indicates larva 1 from family $\mathrm{N}$, clone 8); 4-5 clones were sequenced from each larva (total of 19 clones). ${ }^{\text {h }}$ Mis-spliced, deletion caused by exon skipping. ${ }^{i}$ Mis-spliced, deletion due to alternative $5^{\prime}$ and $3^{\prime}$ splice sites. ${ }^{j}$ Affected codons in the mutant and wild-type sequence (from "Codon" column) do not match because additional mutations occur

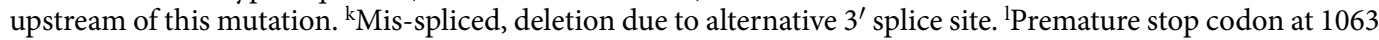
in A1.3 because of deletion upstream of c.3313_3589del. ${ }^{\mathrm{m}}$ Sequence corresponding to insertion not shown due to large number of nucleotide bases. ${ }^{\mathrm{n}} \mathrm{Mis}$-spliced, indel caused by intron retention. ${ }^{\circ}$ Premature stop codon at 1097 in N1.27 and at 1098 in N1.2 and N1.5 because of deletions upstream of c.3418_3589del. PPremature stop codon at 1172 in N1.8 because of deletion upstream of c.3556_3588delins.

from India ${ }^{28}$. Thus, mutations disrupting $P g A B C A 2$ are the primary, but not the only, mechanism of resistance to Cry2 Ab in pink bollworm.

The recessive mutation $r_{A 1}$, which introduces a premature stop codon in $P g A B C A 2$, was fixed in Bt4-R2 in the current and previous study ${ }^{28}$, but was not detected in BX-R. Nonetheless, BX-R harbored other mutations disrupting PgABCA2. For six of the 20 families from single-pair crosses between BX-R and Bt4-R2, larval survival at $10 \mu \mathrm{g}$ Cry $2 \mathrm{Ab}$ per $\mathrm{mL}$ diet was $>99 \%$, implying the parents of these families from both strains had resistance alleles at the same locus (i.e., $P g A B C A 2$ ). However, for five of the 20 single-pair families, larval survival was less than $70 \%$ at the same toxin concentration. In light of the $>92 \%$ survival of each strain at this toxin concentration and the high frequency of $r_{A 1}$ in Bt4-R2, the $<70 \%$ survival in five of the 20 single-pair families implies that mutations at one or more loci other than $P g A B C A 2$ contributed to Cry $2 \mathrm{Ab}$ resistance in BX-R.

Moreover, in contrast with all results from Bt4-R2 and previous results from BX-R ${ }^{25}$, resistance of $B X-R$ to $\mathrm{Cry} 2 \mathrm{Ab}$ was not entirely autosomal or recessive in the current study. We previously reported that BX-R resistance to Cry $2 \mathrm{Ab}$ was autosomal and completely recessive $(h=0)$ at concentrations of 3 and $10 \mu \mathrm{g}$ Cry $2 \mathrm{Ab}$ per mL $\operatorname{diet}^{25}$. Here, mean survival was $25 \%$ higher for $\mathrm{F}_{1}$ progeny from crosses between BX-R and susceptible strain APHIS-S with BX-R fathers than BX-R mothers, indicating non-autosomal inheritance of resistance. In this study, mean values of $h$ for BX-R were 0.82 at $3 \mu \mathrm{g}$ Cry $2 \mathrm{Ab}$ per $\mathrm{mL}$ diet and 0.35 at $10 \mu \mathrm{g}$ Cry $2 \mathrm{Ab}$ per mL diet, indicating non-recessive inheritance of resistance.

We hypothesize that the differences between the current versus previous results with BX-R reflect changes that occurred during the decade ( $>100$ generations) between the two studies. Given that the mutations in PgABCA2 confer autosomal, recessive resistance to Cry2 Ab, we infer this was the primary mechanism of resistance in BX-R at the time of the previous study ${ }^{25}$. Unfortunately, we do not have the preserved samples required to test this hypothesis. We also hypothesize that after the previous study, the frequency of non-autosomal, non-recessive resistance increased in BX-R. The one or more non-autosomal, non-recessive mechanisms of resistance to 
Cry2Ab in BX-R remain to be determined, but probably involve something other than reduced toxin binding, which is typically inherited as a recessive trait ${ }^{13,40}$.

The results here for BX-R resistance to Cry2Ab have some striking parallels with resistance to Cry1Ac in the field-selected R strain of Plutella xylostella from the Philippines ${ }^{41}$. In the R strain, resistance was associated with reduced binding of Cryl Ac and inheritance of reduced binding was autosomal and recessive. However, as for $\mathrm{BX}-\mathrm{R}$, survival was higher for $\mathrm{F}_{1}$ progeny from crosses with resistant fathers and susceptible mothers than for the reciprocal cross, yet sex ratios of the $\mathrm{F}_{1}$ progeny did not fit expectations for sex linkage. Thus, similar to our results with BX-R, the R strain had an autosomal recessive mechanism of resistance (reduced binding), as well as at least one additional mechanism that was not autosomal, not recessive, and did not fit expectations for either maternal or sex-linked inheritance. In a related study, Petzold-Maxwell et al. $(2012)^{42}$ also reported higher resistance to Cry $3 \mathrm{Bb} 1$ of $\mathrm{F}_{1}$ progeny from crosses with resistant fathers and susceptible mothers relative to the reciprocal cross in a lab-selected strain of western corn rootworm (Diabrotica virgifera virgifera). In this case, because males are heterogametic in Coleoptera (the opposite of Lepidoptera), $\mathrm{F}_{1}$ progeny from crosses with resistant fathers and susceptible mothers are expected to have lower resistance relative to the progeny from the reciprocal cross under sex-linked inheritance. Thus, this represents a third example of non-autosomal inheritance of Bt resistance that does not fit either maternal or sex-linked inheritance.

Together with previous findings ${ }^{28}$, the results here show that many mutations in PgABCA2 occur in Cry2Ab-resistant pink bollworm from BX-R, Bt4-R2, and field-selected populations from India. Here we found 15 different $P g A B C A 2$ mutations in three larvae from BX-R and 22 different mutations in four $\mathrm{F}_{1}$ progeny from crosses between BX-R and Bt4-R2. Collectively, in the current and previous study ${ }^{28}$, we have identified 47 different cDNA mutations in PgABCA2 from the 29 larvae analyzed from BX-R, Bt4-R2, and India.

We found only one of the 47 mutations, c1090_1234del, that was common to Cry2Ab-resistant larvae from BX-R, Bt4-R2 and India. This mutation, which causes complete skipping of exon 6 and introduces a premature stop codon, occurred in two of three BX-R larvae (Table 1), two of 14 Bt4-R2 larvae ${ }^{28}$, and three of four resistant $\mathrm{F}_{1}$ progeny from BX-R X Bt4-R2 (Table 2). This was also the most common mutation in the field-selected Cry2Ab-resistant larvae from India ${ }^{28}$. It occurred in six of eight resistant larvae tested and was found in larvae from all four states of India studied (Andhra Pradesh, Karnataka, Maharashtra and Telangana) ${ }^{28}$. Another mutation, c.3097_3100del, was detected in two of the three larvae from BX-R, one of the four Cry2Ab-resistant $\mathrm{F}_{1}$ progeny from BX-R X Bt4-R2, and in one larva from India (KT-1, mislabeled as c.3098_3101del in Mathew et al. $2018^{28}$ ), but not in Bt4-R2. We found each of the other 45 mutations only in one of the three sources of Cry2Ab-resistant pink bollworm we studied: BX-R, Bt4-R2 or India.

Overall, 59\% (28 of 47) of the cDNA mutations identified in PgABCA2 introduce a premature stop codon, including all three mutations mentioned above. Mis-splicing of PgABCA2 pre-mRNA is implicated in eight of the 15 different mutations we found in BX-R (Table 1), including both of the mutations mentioned above that were also found in field-selected larvae from India. Mis-splicing is also involved in all eight mutations previously analyzed from Bt4-R $2^{28}$, four of the ten mutations in $\mathrm{F}_{1}$ progeny from BX-R X Bt4-R2 not identified from previous analyses (Table 2), and in five of the six mutations analyzed from India ${ }^{28}$. Overall, we estimate mis-splicing is involved in $61 \%$ ( 22 of 36) of the cDNA mutations in PgABCA2 characterized here and previously ${ }^{28}$.

Similar to the results showing a prominent role of mis-splicing and diverse mutations disrupting $P g A B C A 2$ in pink bollworm resistance to Cry2 Ab in both lab-and field-selected resistance to Cry2 Ab, mis-splicing and diverse mutations disrupting the cadherin gene $\mathrm{PgCad} 1$ are important in lab- and field-selected pink bollworm resistance to Cry1 Ac ${ }^{32,37-39,43,44}$. In field populations from China that have remained mostly susceptible to Cry1Ac, the most common PgCad1 resistance allele $(r 1)$ was first identified from a lab-selected strain in the United States ${ }^{32}$. However, unlike the two deletions in PgABCA2 (c1090_1234del and c.3098_3101del) associated with Cry2Ab resistance that are shared between field-selected populations from India and one or both lab-selected strains from the United States, none of the specific PgCad1 mutations in field-selected populations from India were detected in lab-selected strains from the U.S. and China ${ }^{32,44}$. For H. armigera from China, mutations in $\mathrm{HaCad}$ and a tetraspanin gene (HaTSPAN1) linked with resistance to Cry1Ac in lab-selected strains also occur in field-selected populations where early warning of resistance has been reported ${ }^{15,45}$.

Aside from pink bollworm in India, the only other currently documented case of practical resistance to Cry2Ab involves Helicoverpa zea in the United States ${ }^{9,46,47}$. It will be intriguing to determine if this resistance is caused by mutations affecting $A B C A 2$, other mechanisms, or both.

\section{Methods}

Insects. We used three strains of pink bollworm that originated from the southwestern United States: APHIS-S, Bt4-R2, and BX-R. APHIS-S is a susceptible strain that had been reared in the laboratory for more than 30 years without exposure to Bt toxins or insecticides ${ }^{48,49}$. Bt4-R2 was obtained from the Bt4R strain in 2010 and had $>10,000$-fold resistance to Cry2Ab relative to APHIS-S that is associated with a mutation in and mis-splicing of $P g A B C A 2^{27,28}$. BX-R was started in 2006 by pooling 875 pupae from substrains BX-R1 and BX-R2, which had been selected in the laboratory with $\mathrm{Cry} 2 \mathrm{Ab}^{25,50}$ and had 210- to 240 -fold resistance to Cry2Ab relative to APHIS-S ${ }^{25,51}$. Larvae were fed wheat germ diet ${ }^{52}$. We conducted rearing, crosses and bioassays at $26^{\circ} \mathrm{C}, 14 \mathrm{~h}$ light: $10 \mathrm{~h}$ dark. For several years before this study, we selected resistance in Bt4-R2 and BX-R by rearing larvae on diet containing $3 \mu \mathrm{g}$ Cry $2 \mathrm{Ab}$ and $10 \mu \mathrm{g}$ Cry1 Ac per mL diet approximately every six generations.

Bt toxin. We used Cry2A.127, an engineered variant of Cry2Ab, that was prepared as described previously ${ }^{53}$ and provided by DuPont Pioneer (now Corteva Agriscience) as a purified and solubilized protoxin. Cry2A.127 shares $98.6 \%$ amino acid sequence identity with Cry2Ab1 and Cry2Ab2 (9 substitutions out of 633 amino acids) and is referred to here as Cry $2 \mathrm{Ab}$. 
Diet Bioassays. We used diet incorporation bioassays to evaluate susceptibility to Cry2 $\mathrm{Ab}^{25,36,54}$. Individual neonates were placed in wells of bioassay trays (BIO-BA-128, Pitman, NJ) containing $\sim 1 \mathrm{~g}$ of diet and covered with Pull N' Peel tab tray covers (BIO-CU-16, Pitman, NJ). We used 0 (control), 3, or $10 \mu \mathrm{g}$ Cry2Ab per mL diet. We scored live fourth instars, pupae, and adults as survivors after 21 and 18 days in the first and second set of bioassays from mass crosses, respectively. We used the shorter duration in the second set to facilitate preservation of survivors before they emerged as moths. Survival at $10 \mu \mathrm{g}$ Cry2Ab per mL diet did not differ significantly between the first and second set of bioassays for each strain or group of $\mathrm{F}_{1}$ progeny (Fisher's exact test, $\mathrm{P}>0.54$ for each comparison).

Dominance, Sex Linkage, and Maternal Effects. To evaluate dominance, sex linkage, and maternal effects of resistance to Cry2Ab in Bt4-R2 and BX-R, we used two sets of bioassays with larvae produced by mass crosses. We started the first and second sets of bioassays in February and March 2015, with the $\mathrm{F}_{45}$ and $\mathrm{F}_{46}$ generations of Bt4-R2 and the $\mathrm{F}_{84}$ and $\mathrm{F}_{85}$ generations of BX-R, respectively. In the generation immediately preceding the first set of bioassays, we had selected both strains on diet containing $3 \mu \mathrm{g}$ Cry $2 \mathrm{Ab}+10 \mu \mathrm{g}$ Cry $1 \mathrm{Ac}$ per mL diet. In both sets of bioassays, we tested $\mathrm{F}_{1}$ progeny from the following seven mass crosses: three within-strain crosses (APHIS-S, Bt4-R2, and BX-R) and four reciprocal crosses between each resistant strain and the susceptible strain

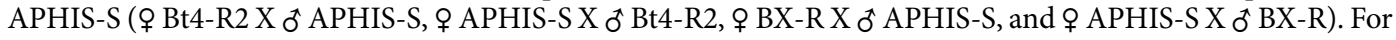
each mass cross, we put 30 pupae of each sex in paper cups $(350 \mathrm{~mL})$ and allowed adults to emerge in the cups, mate, and oviposit ${ }^{27}$. In the first set of bioassays, we tested 32 larvae from the $\mathrm{F}_{1}$ progeny of each mass cross at 0 (control), 3 or $10 \mu \mathrm{g}$ Cry $2 \mathrm{Ab}$ per $\mathrm{mL}$ diet. In the second set of bioassays, we tested 16 larvae from the $\mathrm{F}_{1}$ progeny of each mass cross at 0 or $10 \mu \mathrm{g}$ Cry $2 \mathrm{Ab}$ per $\mathrm{mL}$ diet (total $\mathrm{n}$ for both sets $=872$ ).

Interstrain Complementation Test for Allelism. To determine if the locus or loci conferring resistance to Cry2 Ab differed between Bt4-R2 and BX-R, we performed interstrain complementation tests for allelism ${ }^{54-56}$. We conducted the single-pair crosses and associated bioassays simultaneously with the second set of mass crosses and bioassays described above. In each of 40 plastic cups $(30 \mathrm{~mL}$ plastic Solo cups, Dart Container Cooperation, Mason, MI), we put one female Bt4-R2 pupa and one male BX-R pupa. In each of another 40 cups, we put one female BX-R pupa and one male Bt4-R2 pupa. After adults eclosed, lids were replaced with new ones containing a vial of $15 \%$ sucrose and paper for oviposition pinned inside. We tested $F_{1}$ progeny from 10 families from each of the two reciprocal crosses on control diet without Cry2 Ab (12-16 neonates per family) or on diet with $10 \mu \mathrm{g}$ Cry2Ab per mL diet (17-32 neonates per family). After 18 days, we recorded survival and collected all surviving $4^{\text {th }}$ instars and pupae.

Statistical Analyses of Bioassay Data. Adjusted Survival. For all bioassays, we calculated adjusted survival (\%) as the survival (\%) for a strain or group of $\mathrm{F}_{1}$ progeny tested on treated diet divided by the survival (\%) for the same strain or group of $\mathrm{F}_{1}$ progeny tested simultaneously on untreated (control) diet. All values for survival reported in Results and used for analyses reflect adjusted survival. Mean survival on control diet was 78.1\% $(\mathrm{SE}=3.4 \%)$ and $88.4 \%(\mathrm{SE}=2.9 \%)$ in the first and second sets, respectively.

Sex Linkage and Maternal Effects. To evaluate sex linkage and maternal effects influencing resistance to Cry2Ab, we compared survival of $\mathrm{F}_{1}$ progeny between the two reciprocal crosses with APHIS-S for Bt-R2 and separately for $\mathrm{BX}-\mathrm{R}^{48,54}$. If no significant difference occurs between the reciprocal crosses for a resistant and susceptible strain, inheritance of resistance is considered autosomal, with no evidence of sex linkage or maternal effects. Maternal effects can cause higher survival for the progeny from resistant mothers and susceptible fathers than from susceptible mothers and resistant fathers. Conversely, for Lepidoptera (but not most other insects), sex-linked inheritance of resistance can cause higher survival for the progeny from resistant fathers and susceptible mothers than from resistant mothers and resistant fathers. This is because, for the sex chromosome in Lepidoptera (but not most other insects), females are heterogametic (ZW) and males are homogametic $(\mathrm{ZZ})^{57}$. This means that for sex-linked resistance, females are hemizygotic and carry only one allele at each locus affecting resistance, whereas males carry two such alleles.

With sex linkage inheritance of resistance, the genotypes for a resistant strain can be written as $Z^{r} \mathrm{~W}$ for females and $Z^{r} Z^{r}$ for males; genotypes for a susceptible strain as $Z^{\text {s } W}$ for females and $Z^{\text {s }} Z^{\text {s }}$ for males ${ }^{41}$. Thus, crosses between resistant mothers $\left(Z^{r} \mathrm{~W}\right)$ and susceptible fathers $\left(Z^{s} Z^{s}\right)$ produce $Z^{s} W$ daughters and $Z^{r} Z^{s}$ sons. Crosses between resistant fathers $\left(Z^{r} Z^{r}\right)$ and susceptible mothers $\left(Z^{s} W\right)$ produce $Z^{r} W$ daughters and $Z^{r} Z^{s}$ sons. Because survival is expected to be higher for $Z^{r} W$ than $Z^{s} W$ daughters, higher survival on treated diet is expected in the progeny from crosses with resistant fathers than with resistant mothers. Also, because of the low expected survival of $\mathrm{Z}^{\mathrm{s}} \mathrm{W}$ daughters on treated diet, the proportion of males is expected to be higher in the crosses with resistant mothers than with resistant fathers.

Dominance. We evaluated the dominance parameter $h$, which varies from 0 for completely recessive resistance to 1 for completely dominant resistance. We calculated $h$ for Bt4-R2 and BX-R as: $h=\left(w_{12}-w_{22}\right) /\left(w_{11}-w_{22}\right)$, where $w_{11}, w_{12}$, and $w_{22}$ are the fitnesses at a particular toxin concentration for resistant homozygotes, heterozygotes, and susceptible homozygotes, respectively ${ }^{58}$. As typically done, we applied this method by assuming survival in bioassays is proportional to fitness, the resistant $(R)$ strain is homozygous for resistance, the susceptible $(S)$ strain is homozygous for susceptibility, and their $\mathrm{F}_{1}$ progeny are heterozygotes, which yields: $h=\left(\mathrm{F}_{1}\right.$ survival $-S$ survival)/( $R$ survival $-S$ survival), where the $S$ strain is APHIS-S, and the $R$ strain is either Bt4-R2 or BX-R.

Sex Determination of $F_{1}$ Survivors from Mass Cross Bioassays. Because male fourth instar pink bollworm larvae have purple or dark brown colored testis visible through the dorsal side of their cuticle at $\sim 6-7^{\text {th }}$ 
segment ${ }^{59}$, we determined the sex of preserved $\mathrm{F}_{1}$ progeny from mass crosses using a dissecting microscope. For specimens for which testis were not observed, larvae were dissected to confirm they lacked testis and/or possessed female reproductive tissue (e.g. ovaries). For samples preserved as pupae, we used the methods by Butt and Cantu $(1962)^{60}$ for sex determination.

Allele-Specific PCR of gDNA. We used PCR genotyping of gDNA adapted from Mathew et al. $(2018)^{28}$ to determine whether survivors on $10 \mu \mathrm{g}$ Cry2A.127 per $\mathrm{mL}$ diet from 20 single-pair families from BX-R X Bt4-R2 (Fig. 2) had 0, 1 or 2 copies of the $r_{A 1}$ mutation at the PgABCA2 locus. We extracted gDNA of three survivors from 19 of the 20 families and the only two survivors from family $\mathrm{O}$ (total $\mathrm{n}=59$ ). We used $0.1-0.6 \mu \mathrm{g}$ gDNA as template for PCR with primers $\mathrm{r}_{\mathrm{A} 1}-\mathrm{F}$ and $\mathrm{r}_{\mathrm{A} 1}-\mathrm{R}$. PCR amplicons were separated on $4 \%$ agarose gels containing $1 \mathrm{X}$ SYBR Safe DNA Gel Stain (Thermo Fisher Scientific, Waltham, MA) and checked for the 305-bp $r_{A 1}$ fragment and the 343-bp wild-type fragment. We performed a second genotyping PCR on each gDNA sample using primers $\mathrm{r}_{\mathrm{A1}}-\mathrm{F} 2$ and $\mathrm{r}_{\mathrm{A1}}-\mathrm{R}$ (Supplementary Table S4). These amplify only the wild-type sequence in this region of PgABCA2 because $\mathrm{r}_{\mathrm{A} 1}-\mathrm{F} 2$ corresponds to a wild-type sequence that is missing in alleles with the $r_{A 1}$ mutation.

cDNA Cloning. For cDNA cloning of $P g A B C A 2$, we used three $4^{\text {th }}$ instar survivors on $3 \mu g$ Cry $2 \mathrm{Ab}$ per $\mathrm{mL}$ diet from the BX-R strain as well as two $4^{\text {th }}$ instar $\mathrm{F}_{1}$ survivors on $10 \mu \mathrm{g}$ Cry $2 \mathrm{Ab}$ per $\mathrm{mL}$ diet from two single-pair cross families. Total RNA was extracted using TRI Reagent (Thermo Fisher Scientific) and treated with DNase I (Thermo Fisher Scientific). cDNA was prepared using $1 \mu \mathrm{g}$ of total RNA with both random hexamer primers and oligo-dT primers with a SuperScript IV First-Strand cDNA synthesis kit (Thermo Fisher Scientific) according to the manufacturer's instruction. PgABCA2 cDNA was amplified in PCR using Platinum SuperFi Green PCR Master Mix (Thermo Fisher Scientific) DNA polymerase and $1 \mu \mathrm{M} 163$ pgABCA2-5 and 166pgABCA2-3 oligonucleotide primers (Supplementary Table S4) at: $98^{\circ} \mathrm{C}$ for $30 \mathrm{~s}$ ( 1 cycle); 40 cycles of $98^{\circ} \mathrm{C}$ for $10 \mathrm{~s}, 50.9^{\circ} \mathrm{C}$ for $10 \mathrm{~s}$ and $72^{\circ} \mathrm{C}$ for $3 \mathrm{~min}$; then $72^{\circ} \mathrm{C}$ for $5 \mathrm{~min}$. PCR products were separated on $1 \%$ agarose gels stained with SYBR Safe (Thermo Fisher Scientific) and viewed using an LED UV Illuminator (Maestrogen, Hsinchu City, Taiwan). Bands were gel-purified using E.Z.N.A. Gel Extraction Kit (Omega Bio-Tek, Norcross, GA) and ligated into pCR-XL2-TOPO (Thermo Fisher Scientific). Plasmids were propagated in TOP10 OneShot Chemically Competent E. coli by growing on LB agar plates containing $50 \mu \mathrm{g}$ per $\mathrm{mL}$ Carbenicillin (Research Products International, Mount Prospect, IL) for $72 \mathrm{~h}$ at room temperature. Colonies were prescreened using PCR and plasmid DNA was purified using a QIAprep Spin MiniPrep kit with a QIAcube system (Qiagen, Hilden, Germany). Inserts were sequenced by Retrogen (San Diego, CA) with primers: M13 reverse, T7, 63PgABCA2-3, 64PgABCA2-5, 65PgABCA2-3, 66PgABCA2-5, 67PgABCA2-3, 68PgABCA2 -5, 69PgABCA2-3, 70PgABCA2-5, 71PgABCA2-3, 72PgABCA2-5, 73PgABCA2-3, 74PgABCA2-5, 75PgABCA2-3, 76PgABCA2-5, 77PgABCA2-5 and 78PgABCA2-5 (Supplementary Table S4). PgABCA2 coding sequences obtained from BX-R and $\mathrm{F}_{1}$ progeny between BX-R X Bt4-R2 are deposited in the GenBank public database (MT152670-MT152697). Mutation names are based on guidelines from the Human Genome Variation Society (http://www.hgvs.org/).

gDNA Cloning. To assess if $P g A B C A 2$ mutations in BX-R, initially identified in cDNA from three BX-R individuals, were caused by mutations in gDNA or in messenger RNA (mRNA), we PCR amplified, cloned, and sequenced corresponding gDNA PCR fragments. We extracted gDNA from $4^{\text {th }}$ instar heads using the Gentra Puregene Tissue Kit (Qiagen) according to the manufacturer's instructions. PgABCA2 gDNA fragments were PCR amplified using the Platinum SuperFi Green PCR Master Mix (Thermo Fisher Scientific) and gene-specific oligonucleotide primers (Supplementary Table S4) designed from exons adjacent to mutations identified from cDNA sequencing (Table 1). PCR products were analyzed by agarose gel electrophoresis. DNA bands were gel-purified and cloned into pCR2.1-TOPO (Thermo Fisher Scientific) and Sanger sequenced as above.

Received: 12 March 2020; Accepted: 22 April 2020;

Published online: 14 May 2020

\section{References}

1. Sanahuja, G., Banakar, R., Twyman, R. M., Capell, T. \& Christou, P. Bacillus thuringiensis: A century of research, development and commercial applications. Plant Biotechnol. J. 9, 283-300, https://doi.org/10.1111/j.1467-7652.2011.00595.x (2011).

2. ISAAA. Global Status of Commercialized Biotech/GM Crops in 2018: Biotech Crops Continue to Help Meet the Challenges of Increased Population and Climate Change. ISAAA Brief No. 54 (ISAAA: Ithaca, NY, 2018).

3. Hutchison, W. D. et al. Areawide suppression of European corn borer with Bt maize reaps savings to non-Bt maize growers. Science 330, 222-225, https://doi.org/10.1126/science.1190242 (2010).

4. Lu, Y., Wu, K., Jiang, Y., Guo, Y. \& Desneux, N. Widespread adoption of Bt cotton and insecticide decrease promotes biocontrol services. Nature 487, 362, https://doi.org/10.1038/nature11153 (2012).

5. Downes, S., Walsh, T. \& Tay, W. T. Bt resistance in Australian insect pest species. Curr. Opin. Insect Sci. 15, 78-83, https://doi. org/10.1016/j.cois.2016.04.002 (2016).

6. National Academies of Sciences, Engineering, and Medicine. Genetically Engineered Crops: Experiences and Prospects. (The National Academies Press, Washington, DC, 2016).

7. Dively, G. P. et al. Regional pest suppression associated with widespread Bt maize adoption benefits vegetable growers. Proc. Natl. Acad. Sci USA 115, 3320-3325, https://doi.org/10.1073/pnas.1720692115 (2018).

8. Tabashnik, B. E. \& Carrière, Y. Surge in insect resistance to transgenic crops and prospects for sustainability. Nat. Biotechnol. 35, 926-935, https://doi.org/10.1038/nbt.3974 (2017).

9. Tabashnik, B. E. \& Carrière, Y. Global patterns of resistance to Bt crops highlighting pink bollworm in the United States, China and India. J. Econ. Entomol. 112, 2513-2523, https://doi.org/10.1093/jee/toz173 (2019).

10. Calles-Torrez, V. et al. Field-evolved resistance of northern and western corn rootworm (Coleoptera: Chrysomelidae) populations to corn hybrids expressing single and pyramided Cry3Bb1 and Cry34/35Ab1 Bt proteins in North Dakota. J. Econ. Entomol. 112, 1875-1886, https://doi.org/10.1093/jee/toz111 (2019).

11. Smith, J. L., Farhan, Y. \& Schaafsma, A. W. Practical resistance of Ostrinia nubilalis (Lepidoptera: Crambidae) to Cry1F Bacillus thuringiensis maize discovered in Nova Scotia, Canada. Sci. Rep. 9, 18247, https://doi.org/10.1038/s41598-019-54263-2 (2019). 
12. Wu, Y. D. Detection and mechanisms of resistance evolved in insects to Cry toxins from Bacillus thuringiensis. Adv. Insect Physiol. 47, 297-342, https://doi.org/10.1016/B978-0-12-800197-4.00006-3 (2014).

13. Peterson, B., Bezuidenhout, C. C. \& Van den Berg, J. An overview of mechanisms of Cry toxin resistance in lepidopteran insects. J. Econ. Entomol. 110, 362-377, https://doi.org/10.1093/jee/tow310 (2017).

14. de Bortoli, C. P. \& Jurat-Fuentes, J. L. Mechanisms of resistance to commercially relevant entomopathogenic bacteria. Curr. Opin. Insect Sci. 33, 56-62, https://doi.org/10.1016/j.cois.2019.03.007 (2019).

15. Jin, L. et al. Dominant point mutation in a tetraspanin gene associated with field-evolved resistance of cotton bollworm to transgenic Bt cotton. Proc. Natl. Acad. Sci USA 115, 11760-11765, https://doi.org/10.1073/pnas.1812138115 (2018).

16. Boaventura, D. et al. Molecular characterization of Cry1F resistance in fall armyworm, Spodoptera frugiperda from Brazil. Insect Biochem. Mol. Biol. 116, 103280, https://doi.org/10.1016/j.ibmb.2019.103280 (2020).

17. Carrière, Y., Crickmore, N. \& Tabashnik, B. E. Optimizing pyramided transgenic Bt crops for sustainable pest management. Nat. Biotech. 33, 161-168 (2015).

18. Caccia, S. et al. Binding site alteration is responsible for field-isolated resistance to Bacillus thuringiensis Cry2A insecticidal proteins in two Helicoverpa species. PLoS ONE 5, e9975, https://doi.org/10.1371/journal.pone.0009975 (2010).

19. Tay, W. T. et al. Insect resistance to Bacillus thuringiensis toxin Cry2Ab is conferred by mutations in an $A B C$ transporter subfamily A protein. PLoS Genet. 11, e1005534, https://doi.org/10.1371/journal.pgen.1005534 (2015).

20. Wang, J. et al. CRISPR/Cas9 mediated genome editing of Helicoverpa armigera with mutations of an ABC transporter gene HaABCA2 confers resistance to Bacillus thuringiensis Cry2A toxins. Insect Biochem. Mol. Biol. 87, 147-153, https://doi.org/10.1016/j. ibmb.2017.07.002 (2017).

21. Yang, X. et al. Mutation of $\mathrm{ABC}$ transporter ABCA2 confers resistance to Bt toxin Cry2Ab in Trichoplusia ni. Insect Biochem. Mol. Biol. 112, 103209, https://doi.org/10.1016/j.ibmb.2019.103209 (2019).

22. $\mathrm{Li}$, X. et al. ATP-binding cassette subfamily A member 2 is a functional receptor for Bacillus thuringiensis Cry2A toxins in Bombyx mori, but not for Cry1A, CrylC, Cry1D, Cry1F, or Cry9A toxins Toxins. 12, 104, https://doi.org/10.3390/toxins12020104 (2020).

23. Henneberry, T. J. \& Naranjo, S. E. Integrated management approaches for pink bollworm in the southwestern United States. Integr. Pest Manage. Rev. 3, 31-52, https://doi.org/10.1023/A:1009673622862 (1998).

24. CABI Invasive Species Compendium. Pectinophora gossypiella (pink bollworm). https://www.cabi.org/isc/datasheet/39417 (2020).

25. Tabashnik, B. E. et al. Asymmetrical cross-resistance between Bacillus thuringiensis toxins Cry1 Ac and Cry2Ab in pink bollworm. Proc. Natl. Acad. Sci. USA 106, 11889-11894, https://doi.org/10.1073/pnas.0901351106 (2009).

26. Tabashnik, B. E., Brévault, T. \& Carrière, Y. Insect resistance to Bt crops: Lessons from the first billion acres. Nat. Biotech. 31, 510-521, https://doi.org/10.1038/nbt.2597 (2013).

27. Fabrick, J. A. et al. Multi-toxin resistance enables pink bollworm survival on pyramided Bt cotton Sci. Rep. 5, 16554, https://doi. org/10.1038/srep16554 (2015)

28. Mathew, L. G. et al. ABC transporter mis-splicing associated with resistance to Bt toxin Cry2Ab in laboratory- and field-selected pink bollworm. Sci. Rep. 8, 13531, https://doi.org/10.1038/s41598-018-31840-5 (2018).

29. Tabashnik, B. E. et al. Suppressing resistance to Bt cotton with sterile insect releases. Nat. Biotechnol. 28, 1304-1307, https://doi. org/10.1038/nbt.1704 (2010).

30. USDA Press Release 0222.18, https://www.usda.gov/media/press-releases/2018/10/19/usda-announces-pink-bollwormeradication-significantly-saving (2018).

31. Wan, P. et al. Hybridizing transgenic Bt cotton with non-Bt cotton counters resistance in pink bollworm. Proc. Natl. Acad. Sci USA 114, 5413-5418, https://doi.org/10.1073/pnas.1700396114 (2017).

32. Wang, J. et al. Bt resistance alleles in field populations of pink bollworm from China: Similarities with the United States and decreased frequency from 2012 to 2015. Pest Manag. Sci. 76, 527-533, https://doi.org/10.1002/ps.5541 (2020).

33. Dhurua, S. \& Gujar, G. T. Field-evolved resistance to Bt toxin CrylAc in the pink bollworm, Pectinophora gossypiella (Saunders) (Lepidoptera: Gelechiidae), from India. Pest Manag. Sci. 67, 898-903, https://doi.org/10.1002/ps.2127 (2011).

34. Kranthi, K. R. Pink bollworm strikes Bt-cotton. Cotton Statistics \& News 35, 1-6 (2015).

35. Naik, V. C., Kumbhare, S., Kranthi, S., Satija, U. \& Kranthi, K. R. Field-evolved resistance of pink bollworm, Pectinophora gossypiella (Saunders) (Lepidoptera: Gelechiidae), to transgenic Bacillus thuringiensis (Bt) cotton expressing crystal $1 \mathrm{Ac}(\mathrm{Cry} 1 \mathrm{Ac}$ ) and Cry2 $\mathrm{Ab}$ in India. Pest Manag. Sci. 74, 2544-2554, https://doi.org/10.1002/ps.5038 (2018).

36. Liu, Y. B. et al. Effects of Bt cotton and Cry1 Ac toxin on survival and development of pink bollworm (Lepidoptera: Gelechiidae). J. Econ. Entomol. 94, 1237-1242, https://doi.org/10.1603/0022-0493-94.5.1237 (2001a).

37. Wang, L. et al. Resistance to Bacillus thuringiensis linked with a cadherin transmembrane mutation affecting cellular trafficking in pink bollworm from China. Insect Biochem. Mol. Biol. 94, 28-35, https://doi.org/10.1016/j.ibmb.2018.01.004 (2018).

38. Wang, L. et al. Pink bollworm resistance to Bt toxin Cryl Ac associated with an insertion in cadherin exon 20. Toxins 11, 186, https:// doi.org/10.3390/toxins11040186 (2019a).

39. Wang, L. et al. Transposon insertion causes cadherin mis-splicing and confers resistance to Bt cotton in pink bollworm from China. Sci. Rep. 9, 7479, https://doi.org/10.1038/s41598-019-43889-x (2019b).

40. Tabashnik, B. E. et al. Insect resistance to Bacillus thuringiensis: Uniform or diverse? Philos. Trans. R. Soc., B 353, 1751-1756, https:// doi.org/10.1098/rstb.1998.0327 (1998).

41. Martínez-Ramírez, A. C., Escriche, B., Real, M. D., Silva, F. J. \& Ferré, J. Inheritance of resistance to a Bacillus thuringiensis toxin in a field population of diamondback moth (Plutella xylostella). Pestic. Sci. 43, 115-120, https://doi.org/10.1002/ps.2780430204 (1995).

42. Petzold-Maxwell, J. L., Cibils-Stewart, X., French, B. W. \& Gassmann, A. J. Adaptation by western corn rootworm (Coleoptera: Chrysomelidae) to Bt maize: Inheritance, fitness costs, and feeding preference. J. Econ. Entomol. 105, 1407-1418, https://doi. org/10.1603/ec11425 (2012)

43. Morin, S. et al. Three cadherin alleles associated with resistance to Bacillus thuringiensis in pink bollworm. Proc. Natl. Acad. Sci USA 100, 5004-5009, https://doi.org/10.1073/pnas.0831036100 (2003).

44. Fabrick, J. A. et al. Alternative splicing and highly variable cadherin transcripts associated with field-evolved resistance of pink bollworm to Bt cotton in India. PLoS ONE 9(5), e97900, https://doi.org/10.1371/journal.pone.0097900 (2014).

45. Zhang, H. et al. Diverse genetic basis of field-evolved resistance to Bt cotton in cotton bollworm from China. Proc. Natl. Acad. Sci USA 109, 10275-10280, https://doi.org/10.1073/pnas.1200156109 (2012).

46. Bilbo, T. R., Reay-Jones, F. P. F., Reisig, D. D., Greene, J. K. \& Turnbull, M. W. Development, survival, and feeding behavior of Helicoverpa zea (Lepidoptera: Noctuidae) relative to Bt protein concentrations in corn ear tissues. PLoS ONE 14, e0221343, https:// doi.org/10.1371/journal.pone.0221343 (2019).

47. Kaur, G. et al. Field-evolved resistance of Helicoverpa zea (Boddie) to transgenic maize expressing pyramided Cry1A.105/Cry2Ab2 proteins in northeast Louisiana, the United States. J. Invertebr. Pathol. 163, 11-20, https://doi.org/10.1371/journal.pone.0221343 (2019).

48. Liu, Y. B. et al. Genetics of pink bollworm resistance to Bacillus thuringiensis toxin Cry1Ac. J. Econ. Entomol. 94, 248-252, https:// doi.org/10.1603/0022-0493-94.1.248 (2001b).

49. Bartlett, A. C. Resistance of the pink bollworm to Bt transgenic cotton. In Proceedings, Beltwide Cotton Conferences. National Cotton Council of America, Memphis, TN. pp 766-768 (1995).

50. Carrière, Y. et al. Gossypol in cottonseed increases the fitness cost of resistance to Bt cotton in pink bollworm. Crop Prot. 126, 104914, https://doi.org/10.1016/j.cropro.2019.104914 (2019). 
51. Tabashnik, B. E. et al. Efficacy of genetically modified Bt toxins alone and in combinations against pink bollworm resistant to Cry1Ac and Cry2Ab. PLoS ONE 8, e80496, https://doi.org/10.1371/journal.pone.0080496 (2013).

52. Bartlett, A. C. \& Wolf, W. W. Pectinophora gossypiella. Handbook of Insect Rearing. Vol. 2 (eds. Singh, P. \& Moore, R. F.) 415-430 (Elsevier Scientific Publishing Co., Amsterdam, 1985).

53. Liu, L. et al. Identification and evaluations of novel insecticidal proteins from plants of the class Polypodiopsida for crop protection against key lepidopteran pests. Toxins 11,383, https://doi.org/10.3390/toxins11070383 (2019).

54. Fabrick, J. A. \& Tabashnik, B. E. Similar genetic basis of resistance to Bt toxin CrylAc in boll-selected and diet-selected strains of pink bollworm. PLoS ONE 7(4), e35658, https://doi.org/10.1371/journal.pone.0035658 (2012).

55. Tabashnik, B. E. et al. Global variation in the genetic and biochemical basis of diamondback moth resistance to Bacillus thuringiensis. Proc. Natl. Acad. Sci. USA 94, 12780-12785, https://doi.org/10.1073/pnas.94.24.12780 (1997).

56. Gao, M., Wang, X., Yang, Y., Tabashnik, B. E. \& Wu, Y. Epistasis confers resistance to Bt toxin Cryl Ac in the cotton bollworm. Evol. Appl. 11, 809-819, https://doi.org/10.1111/eva.12598 (2018).

57. Traut, W., Sahara, K. \& Marec, F. Sex Chromosomes and sex determination in Lepidoptera. Sex. Dev. 1, 332-346, https://doi. org $/ 10.1159 / 000111765$ (2007).

58. Liu, Y. B. \& Tabashnik, B. E. Inheritance of resistance to the Bacillus thuringiensis toxin Cry1C in the diamondback moth. Appl. Environ. Microbiol. 63, 2218-2223 (1997).

59. Bartlett, A. \& Lewis, L. J. Genetics of the pink bollworm: rust, orange, and garnet eye colors. Ann. Entomol. Soc. Am. 71, 813-816, https://doi.org/10.1093/aesa/71.6.813 (1978).

60. Butt, B. A. \& Cantu, E. Sex Determination of Lepidopterous Pupae. Agricultural Research Service, U.S. Department of Agriculture, ARS-33-75 https://doi.org/10.5962/bhl.title.67261 (1962).

\section{Acknowledgements}

We thank Corteva Agriscience for providing Cry2A.127 used in this study. Mention of trade names or commercial products in this article is solely for the purpose of providing specific information and does not imply recommendation or endorsement by the U.S. Department of Agriculture. USDA is an equal opportunity provider and employer.

\section{Author contributions}

J.A.F. and B.E.T. designed the study. J.A.F., D.M.L., G.C.U., and A.J.Y. performed the experiments. J.A.F., X.L., and B.E.T. analyzed the data. J.A.F., B.E.T., X.L., and Y.C. wrote the manuscript. All authors have read and approved the manuscript for publication.

\section{Competing interests}

This is a cooperative investigation between USDA ARS and the University of Arizona, with J.A.F., Y.C., and B.E.T. receiving funding from USDA National Institute of Food and Agriculture (Agriculture and Food Research Initiative Program Grant \#2018-67013-27821) and Corteva Agriscience (agreement \#58-3K95-41666) to support this work. D.L., G.C.U, and A.J.Y declare no potential conflict of interest. J.A.F. is coauthor of a patent "Cadherin Receptor Peptide for Potentiating Bt Biopesticides" (patent numbers: US20090175974A1, US8354371, WO2009067487A2, WO2009067487A3). B.E.T. is a coauthor of a patent on modified Bt toxins, "Suppression of Resistance in Insects to Bacillus thuringiensis Cry Toxins, Using Toxins that do not Require the Cadherin Receptor" (patent numbers: CA2690188A1, CN101730712A, EP2184293A2, EP2184293A4, EP2184293B1, WO2008150150A2, WO2008150150A3). Bayer Crop Science, BASF, and Syngenta did not provide funding to support this work but may be affected financially by publication of this paper and have funded other work by J.A.F., Y.C., X.L., and/or B.E.T.

\section{Additional information}

Supplementary information is available for this paper at https://doi.org/10.1038/s41598-020-64811-w.

Correspondence and requests for materials should be addressed to J.A.F.

Reprints and permissions information is available at www.nature.com/reprints.

Publisher's note Springer Nature remains neutral with regard to jurisdictional claims in published maps and institutional affiliations.

(c) (i) Open Access This article is licensed under a Creative Commons Attribution 4.0 International (c) License, which permits use, sharing, adaptation, distribution and reproduction in any medium or format, as long as you give appropriate credit to the original author(s) and the source, provide a link to the Creative Commons license, and indicate if changes were made. The images or other third party material in this article are included in the article's Creative Commons license, unless indicated otherwise in a credit line to the material. If material is not included in the article's Creative Commons license and your intended use is not permitted by statutory regulation or exceeds the permitted use, you will need to obtain permission directly from the copyright holder. To view a copy of this license, visit http://creativecommons.org/licenses/by/4.0/.

(C) The Author(s) 2020 